\title{
Application of multiphase flow and droplet separation theory in modeling cough droplets contamination range to mitigate COVID-19 transmission: Do not stand too close to me!
}

\author{
Eissa M. Al-Safran \\ Petroleum Engineering Dept., College of Engineering and Petroleum, Kuwait University, Kuwait \\ Corresponding Author : e.alsafran@ku.edu.kw
}

Submitted : 10/10/2020

Revised : :16/01/2021

Accepted : 26/01/2021

\begin{abstract}
On March 11, 2020, the World Health Organization (WHO) declared that COVID-19 is a pandemic, warning the world of a health catastrophe and social, economic, and political disruptions. According to WHO, COVID-19 is transmitted by the transport of respiratory droplets generated by a violent respiratory event such as sneeze and cough directly to susceptible persons, or indirectly through surfaces. The aim of this study is to propose simple physical and mathematical models based on two-phase flow dynamics and droplet separation theory. The proposed mathematical model predicts the contamination range of ejected cough droplets, estimating the safe person-to-person social distance. As a result, the proposed simple model predicted a contamination range of $2.3 \mathrm{~m}$ for a male adult. In addition, to understand the behavior of ejected cough droplets, a sensitivity analysis is carried out to investigate the effect on contamination range of cough air flowrate, i.e., body/lung size, droplet size, and droplet drag coefficient. It is found that as the body/lung size decreases, i.e., lower cough flow rate, contamination range decreases, resulting in $1.9 \mathrm{~m}$ for an adult female, and $1.4 \mathrm{~m}$ for a child. In addition, the model predictions show an appreciable effect of droplet size, and droplet drag coefficient on cough contamination range. In particular, the effect of droplet drag coefficient is of interest, because of its relationship to ambient conditions such as temperature and relative humidity, in which both affect ambient air viscosity, and thus drag coefficient. This is important in investigating the contamination range and person-to-person social-distance as climate changes.
\end{abstract}

Keywords: COVID-19; Two-phase flow; Droplet separation; Transmission.

\section{INTRODUCTION}

In this introduction, a brief historical layout of the COVID-19 breakout is first presented. The COVID-19 transmission mode is then discussed to answer the question on how the coronavirus is transmitted, explaining its mysterious high transmission rate. Lastly, a brief discussion of cough dynamics is presented, focusing on its multiphase flow behavior. 


\section{Historical Perspective of COVID-19 Pandemic}

In December 2019, a novel type of coronavirus broke out in Wuhan, China, which was not identified previously in humans. On December 31, the WHO was alerted by Chinese health officials of the breakout. WHO then declared that the coronavirus is a global emergency on January 30, after it had spread in other countries. On February 11, 2020, the WHO named the novel coronavirus as COVID-19, by which time China had reported 1,016 deaths and 42,638 infections. Finally, on March 11, 2020, the WHO declared COVID-19 is a pandemic, warning that in short time the virus will spread to all countries, causing catastrophic health, economic, social, and political disruptions to the entire world (Qamar, 2020). As of January 15, 2021, 91.5 million people were infected, and approximately 2 million deaths were confirmed, mainly involving 65+ year old and/or with pre-existing health conditions (https://covid19. who.int/). According to modeling studies by Imperial College in London, if no mitigation measures are taken, COVID-19 will globally infect 7 billion people and kill 40 million people this year (Walker et al., 2020). This indicates that aggressive mitigation and suppression measures must be in place to control the pandemic and save lives.

Historically, the Spanish flu (H1N1) pandemic in 1918-1919 infected one-quarter of the world population and killed 40 million people. In 2002-2004 SARS infected 8,098 people among which 774 died (WHO-2002 reports). The World Bank in 2005 estimated that a pandemic could cost the global economy $\$ 800$ billion per year. This clearly shows the need for scientific understanding and predictive models to predict and control the transmission of pandemics to avoid global social, economic, and political disruptions.

\section{Modes of Transmission of COVID-19}

Understanding the physical processes of COVID-19 transmission is crucial in designing mitigation strategies and control measures. COVID-19 transmits via respiratory fluid droplets laden with infectious pathogens generated from the lungs and respiratory tracts of an infected host to a susceptible person. Knowledge of droplets generation, transport, and deposition/inhalation is the key to modeling the transmission of COVID-19 and determining minimum person-to-person social distance, and basic virus reproduction number (Drossinos and Stilianakis, 2020). Generated respiratory droplets in the human respiratory tract are fragmented and expelled by violent expiratory events, such as cough and sneeze, or gentle events such as breathing, speaking, and laughing. Weber and Stilianakis (2008) defined three transmission modes of pathogens, namely "contact," "droplet," and "airborne." Recently, Asadi et al. (2020) simplified the classification into two categories, namely "contact," and "airborne," each of which is classified into two sub-categories as shown in Fig. 1.

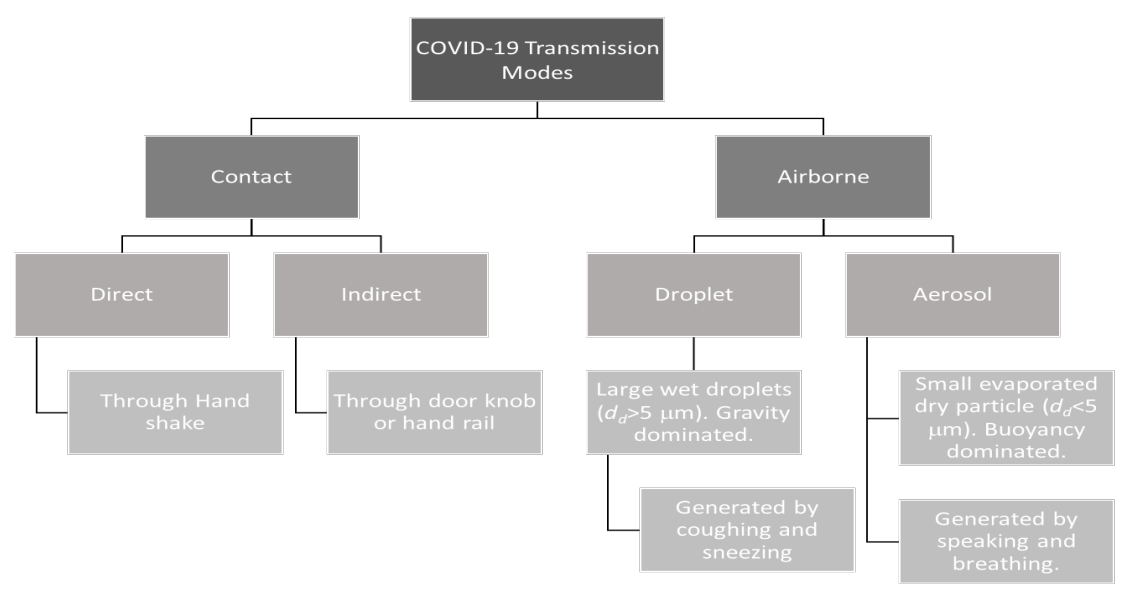

Figure 1. COVID-19 person-to-person transmission modes. 
Contact transmission is classified into direct and indirect contacts for droplets laden with pathogens. Direct contact is the transfer of pathogens via physical touching between an infected host and a susceptible person by, for example, handshake. Conversely, indirect contact is the transfer of pathogens through a surface containing settled droplets, such as currency or goods exchange. The droplet transmission mechanism is the transfer of large saliva and mucus droplets from the mouth of an infected host via turbulent jet airflow generated by an expiratory event such as cough or sneeze. The large liquid droplets are sprayed and then directly inhaled and deposited in the mouth, nose, or eyes of a susceptible host. Aerosol transmission is the transmission of very small respiratory solid droplet nuclei $(<$ $5 \mu \mathrm{m}$ ), which can remain airborne for a long time and transport over long distances through ventilation systems (Hsiao et al, 2020). These droplets are often the result of an evaporation process of large liquid droplets, which exposes the pathogen, thus called "droplet nuclei." The classification of the transmission mode is mainly based on the expelled droplet size, which is quite challenging to measure (Drossinos and Stilianakis, 2020). This study investigates the physics of the large droplet transmission via a cough and proposes a simple mathematical model to predict the range of the expelled droplets.

\section{Cough Dynamics}

Among the respiratory events, coughing is the main respiratory event where the risk of infection is high due to the large size and concentration of expelled liquid droplets (Duguid, 1946). Because coughing is the common symptom for COVID-19, this study is focused on modeling the two-phase mechanism in a cough. A cough is an ejected multiphase turbulent cloud, which consists of dry gas and suspended liquid droplets containing minerals and pathogens as a solid phase. Although the large droplets follow a ballistic trajectory unaffected by the surrounding gas phase, the small droplets evaporate to a dry solid nuclei (pathogen) that are dominated by buoyancy, thus remain suspended for a long period of time and an extending range (Bourouiba, 2020).

The aim of this study is to propose simple physical and mathematical models using two-phase flow dynamics and separation theory to determine cough droplets' contamination range, the required person-to-person distance to control the virus transmission. Beyond the droplets' contamination range, COVID-19 transmission risk is significantly low due to droplets settling under gravity. In the next section, a simple cough physical model is proposed based on experimental observations. Important parameters such as droplet size, cough cross-sectional area, and cough flow rate are discussed, which is followed by the proposed simple mathematical model and calculation scheme. A discussion of the model results is presented next and the effect of cough characteristics and ambient condition is investigated. The paper is closed with a conclusion of the finding of the paper and possible future work.

\section{Physical Model}

A respiratory function such as cough or sneeze is a multiphase flow natural process in which liquid droplets of mucus and saliva are entrained in a relatively hot dry air exhaled from the lungs through the throat and the mouth. As this multiphase mixture leaves the mouth, it undergoes hydrodynamic and thermal processes due to interaction with its surrounding ambient environment, which determines the behavior of the exhaled mixture in space and time.

The multiphase flow mixture of a cough or sneeze contains different sizes of liquid droplets, which can be plausibly classified into two components, namely a homogenous cloud or puff, and ballistic large liquid droplets. Bourouiba et al. 2014 experimentally investigated these components in a sneeze, as shown in Fig. 2. Fig. 2a shows the generated multiphase cloud, which entrains mucosalivary liquid droplets. It also shows that as the cloud flows away from the source, it increases in size, thus decreasing its mean velocity, resulting in droplets settling and falling under the effect of gravity. Fig. 2b shows the ballistic behavior of the large liquid droplets and their downward settling trajectory as they move further from the source. Bourouiba et al. measurements indicated that the settling of the large 
liquid droplets defines the contamination range of the respiratory function. The gas void fraction of the cloud then increases (low liquid concentration), which increases the buoyancy force acting on the cloud, resulting in an upward flow. The buoyancy force increase may also be due a low cloud density because of its high temperature.
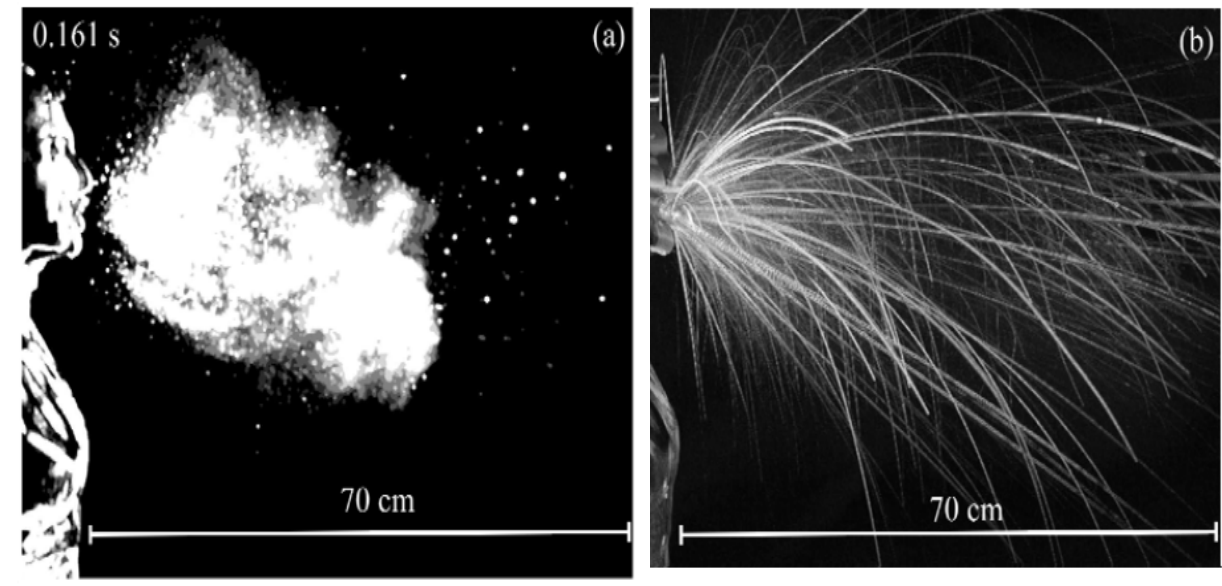

Figure 2. Multiphase flow dynamics during a respiratory event: a) cloud multiphase flow behavior, b) large liquid droplets ballistic trajectories (Bourouiba et al. 2014).

Fig. 3 shows a simplified physical model proposed in this study. This model illustrates that as cough or sneeze is exhaled from a human mouth, the gas/liquid mixture flow takes a conical shape where the flowing cross-sectional area (A) increases with distance (D) from the source as observed by Bourouiba et al. (2014). In the current study, the two-phase flow mixture of a cough or sneeze is postulated to have two regions, namely a no-slip (homogenous) region, and a slip region. The no-slip region is characterized by a small cross-sectional area, high mixture velocity, high droplet concentration, with liquid droplets traveling at the same velocity as the surrounding dry gas. Beyond a distance (D) from the source, i.e. the droplet contamination range, and due to an increase in the cough flowing crosssectional area, several flow mechanisms occur. For example, the large liquid droplets fall down due to a decrease in flow inertia and an increase in gravitational force domination. As the relatively large droplets separate, the gas void fraction of the cough increases, promoting the buoyant force effect on the small droplets and resulting in an upward flow of the cough cloud.
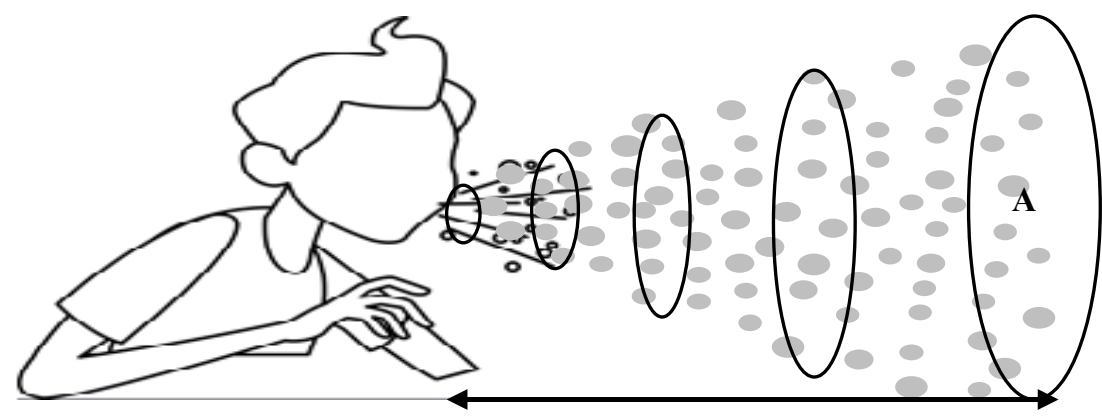

D

Figure 3. Proposed cough physical model. 
The physical model consists of several parameters, which are required as inputs in the proposed mathematical model. For example, average droplet size or droplet size distribution, flow cross-sectional area (flow conical shape dimensions), cough or sneeze dry gas flow rate, and fluid physical properties. In this study, these parameters are obtained from experimental studies found in the literature as presented in the following sections.

\section{Cough Ejected Droplet Size}

Although the ejected droplet size during a cough is the most important parameter in modeling the transmission, it is the most challenging to measure. A single cough generates approximately 100-1000 droplets with a velocity in the range of $10 \mathrm{~m} / \mathrm{s}$ (Asadi et al., 2019), and a Reynolds number of $O\left(10^{4}\right)$ and higher (Bourouiba et al., 2014). The expelled fluid during a cough is initially in the form of fragmented sheets, which breaks up into droplets if the Weber number is sufficiently large (Mittal, et al., 2020).

Several investigators [Duguid (1946), Loudon and Roberts (1967), Papineni and Rosenthal (1997), Chao et al. (2009)] conducted experimental studies to measure the droplet size distribution of different respiratory events, including coughing, sneezing, nose and mouth breathing, and talking. Table 1 shows the droplet size statistical characteristics in these studies, including their measurement techniques. Specifically, Fig. 4 shows the droplet diameter distribution of a cough measured by Duguid (1946). The distribution is clearly Inverse-Gaussian, with a mode of approximately $16 \mu \mathrm{m}$. The Wei and Li (2017) experimental study of a real and simulated cough revealed that realistic droplet sizes in a cough ranges between 8-14 $\mu \mathrm{m}$ for small particles, 57-68 $\mu \mathrm{m}$ for medium particles, and $96-114 \mu \mathrm{m}$ for large particles, which all fall within the Duguid (1946) droplet size distribution. As an input for the proposed model, the Duguid mode value of $16 \mu \mathrm{m}$ is selected.

Table 1. Droplet sizes of different respiratory events (Reproduced from Aliabadi et al., 2011).

\begin{tabular}{|l|c|l|c|c|c|c|}
\hline Study & $\begin{array}{c}\text { Measurement } \\
\text { technique }\end{array}$ & Expiration type & $d_{\text {min }}\left(\mu_{m}\right)$ & $d_{\text {max }}\left(\mu_{m}\right)$ & $\begin{array}{c}\text { Geometric } \\
\text { mean }\left(\mu_{m}\right)\end{array}$ & $\begin{array}{c}\text { Geometric standard } \\
\text { deviation }\left(\mu_{m}\right)\end{array}$ \\
\hline Duguid (1946) & Microscopy & Coughing & 1 & 2000 & 14 & 2.6 \\
\hline Duguid (1946) & Microscopy & Sneezing & 1 & 2000 & 8.1 & 2.3 \\
\hline Loudon and Roberts (1967) & Microscopy & Coughing & 1 & $>1471$ & 12 & 8.4 \\
\hline Papineni and Rosenthal (1997) & OPC & Talking & $<0.6$ & 2.2 & 0.8 & 1.5 \\
\hline Papineni and Rosenthal (1997) & OPC & Nose breathing & $<0.6$ & 2.2 & 0.8 & 1.5 \\
\hline Papineni and Rosenthal (1997) & OPC & Mouth breathing & $<0.6$ & 2.5 & 0.7 & 1.4 \\
\hline Papineni and Rosenthal (1997) & OPC & Coughing & $<0.6$ & 2.5 & 0.7 & 1.5 \\
\hline Papineni and Rosenthal (1997) & ATEM & Mouth breathing & $<0.6$ & 2.5 & 1.2 & 1.6 \\
\hline Chao et al. (2009) & IMI & Talking & 2 & 2000 & 12.6 & 3.2 \\
\hline Chao et al. (2009) & IMI & Coughing & 2 & 2000 & 13.1 & 3.6 \\
\hline OPT: optical particle counter. ATEM: analytical transmission electron microscope. IMl: interferometric Mie imaging \\
\hline
\end{tabular}




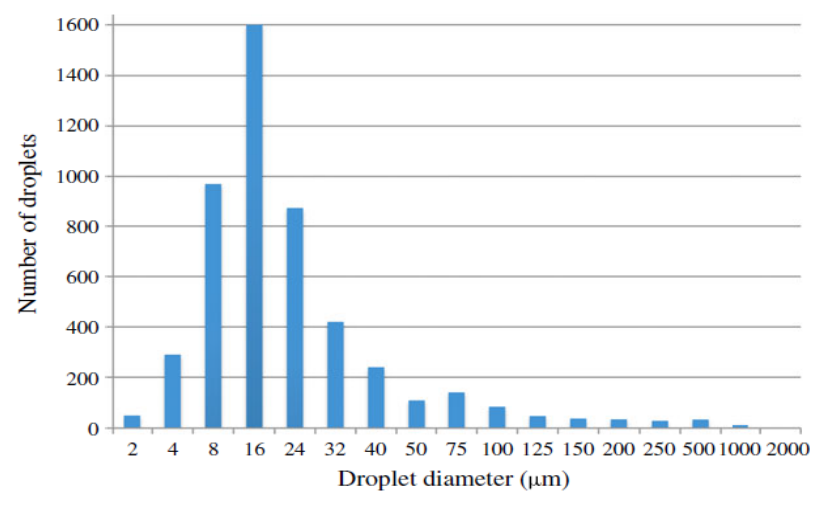

Figure 4. Cough droplet size distribution (Duguid, 1946).

\section{Cough Cross-Sectional Area Profile}

Wei and Li (2017) experimental images of real and simulated coughs revealed a cone-shape profile of cough flow cross-sectional area as shown in Fig. 5. The cross-sectional area begins from the mouth opening area and increases linearly with distance from the source, indicating a decrease of cough cloud mean velocity.

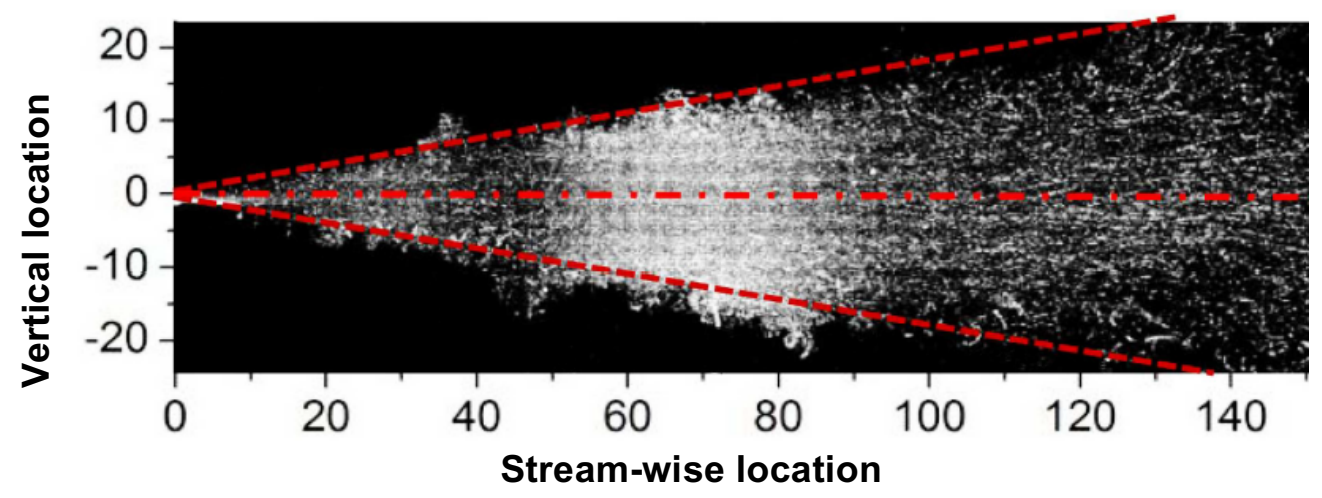

Figure 5. Cough particles contamination shape and profile (Wei and Li, 2017).

To quantify the cone-shaped profile of the cough cross-sectional area, Bourouiba et al. (2014) experimentally investigated the characteristics of analog expiratory events, including the cough/sneeze cloud conical shape profile. Their measured experimental profile is a relationship between the distance travelled by the cough/sneeze cloud geometric center and the mean radius of the cough/sneeze cloud for different experimental conditions. In this study, using their data, a relationship between the cough/sneeze cloud conical shape cross-sectional area and traveled distance is developed and correlated. This was found to be a linear relationship, as shown in Fig. 6. In this study, a simple linear regression model is fitted to the data and the simple linear regression model in Eq. 1 is developed, which has a coefficient of variation $\left(\mathrm{R}^{2}\right)$ of 0.82 .

$$
A_{c}=1.61 D+2.94
$$


where $A_{c}=$ cough cross-sectional area in $\mathrm{cm}^{2}$, and $D=$ distance from source in $\mathrm{cm}$. Eq. 1 is used as input to the proposed model to calculate the actual droplet velocity.

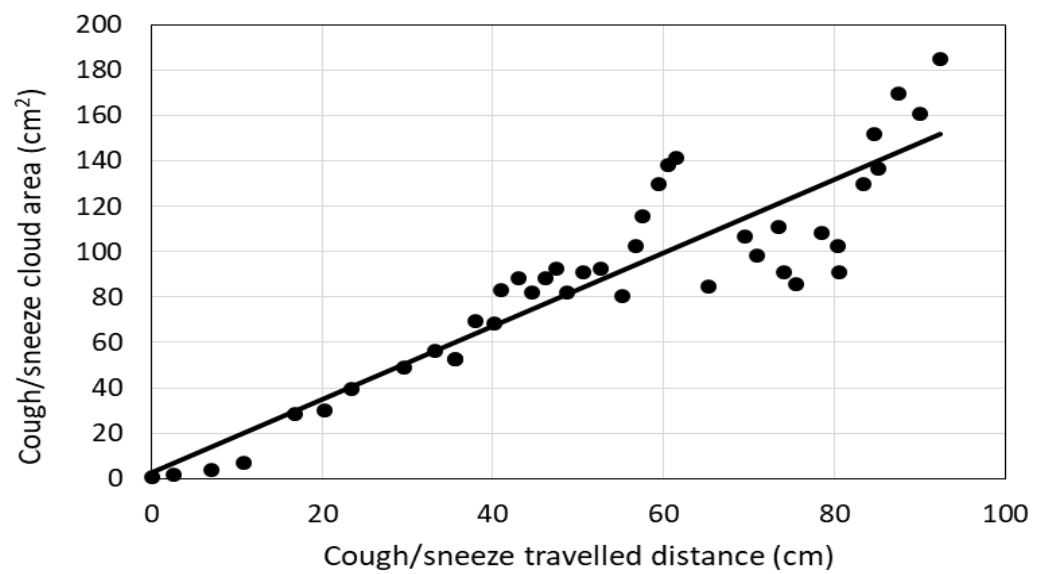

Figure 6. Relationship between cough/sneeze cross-sectional area and travel distance.

\section{Cough Flow Rate}

Several experimental studies have been carried out to characterize a cough and its three main characteristics, namely Cough Peak Flow Rate (CPFR), Cough Expired Volume (CEV), and Peak Velocity Time (PVT), which are shown in a typical cough plot given in Fig. 7 (Lamb et al., 1993). Leiner et al. (1966) developed a multiple linear regression model to predict CPFR as a function of age and height of human subjects. In addition, Mahajan et al. (1994) and Singh et al. (1995) correlated CPFR, CEV and PVT for males and females. Zhu et al. (2006) used particle image velocimetry (PIV) to capture the flow field and measured the peak cough velocity, which can be used to calculate the mouth opening area during a cough. Their experimental study revealed an average CEV of 1.4 liter with a range of 0.8-2.2 liters and an average velocity of $11.2 \mathrm{~m} / \mathrm{s}$ with a variation of 6 to $22 \mathrm{~m} / \mathrm{s}$. Measurements by Mahajan et al. (1994) revealed an average CEV of 3 liters with variation of up to 5 liters.

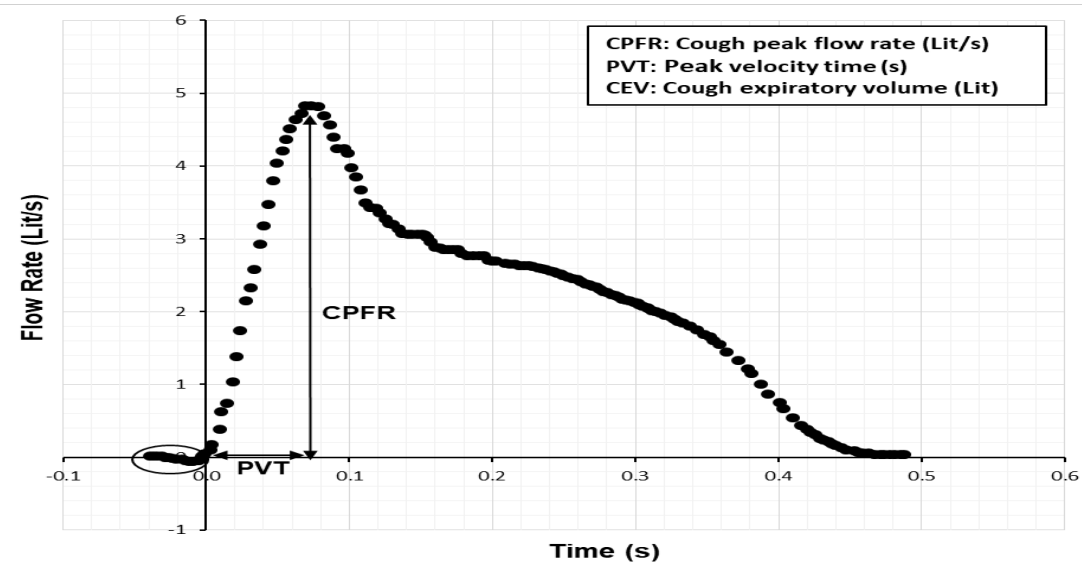

Figure 7. Typical cough characteristics (Reproduced from Gupta et al., 2009). 
Gupta et al. (2009) conducted a comprehensive experimental study to measure and empirically model cough characteristics of 25 male and female subjects. Table 2 shows the range of the measured data of CPFR, CEV, and PVT for both genders.

Table 2. Experimental measured chough characteristics (Gupta et al., 2009).

\begin{tabular}{|l|c|c|}
\hline Parameter & Male & Female \\
\hline CPFR (Lit/s) & $3-8.5$ & $1.6-6$ \\
\hline CEV (mLit) & $400-1600$ & $250-1250$ \\
\hline PVT (msec) & $57-96$ & $57-110$ \\
\hline
\end{tabular}

In the present study, cough characteristics of CPFR, CEV, and PVT are predicted using the Gupta et al. empirical multiple linear regression models for male and female, as are given in Eqs. 2-7. To determine the dimensionless cough average flow rate, Eq. 15 is solved and substituted into Eq. 16 to determine the average cough flow rate. Detailed modeling results are presented below.

\section{THEORETICAL MODELING}

Computational fluid dynamics (CFD) is commonly used to predict the flow behavior and characteristics of respiratory events such as cough or sneeze. However, modeling multiphase turbulence with CFD is challenging due to poor understanding of cough turbulence physics, high computational expense, time consuming in pre- and postprocessing, and required thermal and flow boundary conditions [Zhu et al. (2006), and Zhao et al. (2005)]. Table 3 summarizes the CFD methods typically used for simulating cough and sneeze multiphase turbulence dynamics, indicating their limitations, namely the intense computational power, significant time for convergence, need for accurate boundary conditions and model validation to ensure accuracy and reliability of predictions. Alternately, this study proposes a simplified mechanistic model, which yields reliable results in a very short time and low computational requirements. In addition, the proposed mechanistic model can be improved as the physics and flow dynamics of the phenomena are further understood.

Table 3. Computational fluid dynamics methods (Reproduced from Aliabadi et al., 2011).

\begin{tabular}{|l|c|c|c|c|}
\hline Model & Advantage & Disadvantage & Cells & Time \\
\hline DNS & Resolves eddies of all lengths & Computationally very expensive & $1.00 \mathrm{E}+11$ & Years \\
\hline LES & Resolves large eddies & Computationally expensive & $1.00 \mathrm{E}+09$ & Months \\
\hline DES & Computationally economic & Difficult to implement & $1.00 \mathrm{E}+08$ & Weeks \\
\hline RANS & Computationally economic & Less accurate, difficult to converge & $1.00 \mathrm{E}+07$ & Days \\
\hline DNS: direct numberical sim., LES: Iarge eddy sim., DES: detached eddy sim., RANS: Reynolds ave. Navier-Stokes \\
\hline
\end{tabular}

\section{Cough Flow Rate Empirical Model}

Gupta et al. (2009) developed a set of empirical linear regression models to predict the cough characteristics, namely CPFR, CEV, and PVT for males and females as follows. 
For males:

$$
\begin{aligned}
& C P F R=-8.90+6.39 h+0.0346 w \\
& C E V=0.138(C P F R)+0.2983 \\
& P V T=1.36(C P F R)+65.86
\end{aligned}
$$

For females:

$$
\begin{aligned}
& C P F R=-3.97+4.63 h \\
& C E V=0.204(C P F R)-0.043 \\
& P V T=3.152(C P F R)+64.63
\end{aligned}
$$

where $h=$ height in meter, $w=$ weight in $\mathrm{kg}, C P F R=$ Cough peak flow rate in liters/s, $C E V=$ cough expired volume in liters, and PVT = peak velocity time in ms. Eqs. 2 and 5 show that the CPFR increases as the height and the weight of the subject increases due to the increase of lung size and thus the exhaled air volume. According to Gupta et al. (2009), Eqs. 2-7 are within 20\% uncertainty when compared with their database.

In addition, Gupta et al. (2009) modeling work proposed a Gamma probabilistic model as the best fit for their experimental data. To fit the Gamma distribution model and obtain the model constants, Gupta et al. introduced the following dimensionless parameters:

$$
\begin{aligned}
& \Theta=\frac{q}{C P F R} \\
& \tau=\frac{t}{P V T}
\end{aligned}
$$

where $\Theta=$ dimensionless cough flow rate, $\tau=$ dimensionless time, $q=$ cough flow rate in liters $/ \mathrm{s}$, and $t=$ time in seconds. Because of the asymmetrical cough flowrate distribution before and after the CPFR as shown in Fig. 7, the Gamma probabilistic model used in Gupta et al. has two sets of fitting constants. The first set is for the upward segment of the curve from zero to CPFR $(\tau<1.2)$, and the second is for the downward segment of the curve from 1.2 onward ( $\tau \geq 1.2$ ). The Gamma probability models for $\tau<1.2$ is given as follows:

$$
\Theta_{1}=\frac{a_{1} \tau^{b_{1}-1} \exp \left(-\tau / c_{1}\right)}{\Gamma\left(b_{1}\right) c_{1}^{b_{1}}}
$$

where $\mathrm{a}_{1}=1.68, \mathrm{~b} 1=3.338$, and $\mathrm{c} 1=0.428$. Using these constants, the Gamma probabilistic model mean and variance are defined as $\mu=b_{1} / a_{1}$ and $\sigma^{2}=b_{1} / a_{1}^{2}$, respectively. The Gamma probability for $\tau \geq 1.2$ is given as follows: 


$$
\Theta_{2}=\frac{a_{2}(\tau-1.2)^{b_{2}-1} \exp \left[-(\tau-1.2) / c_{2}\right]}{\Gamma\left(b_{2}\right) c_{2}^{b_{2}}}
$$

where $\mathrm{a}_{2}, \mathrm{~b}_{2}$, and $\mathrm{c}_{2}$ are functions of the cough characteristics and given as

$$
\begin{aligned}
& a_{2}=\frac{C E V}{(P V T)(C P F R)}-a_{1} \\
& b_{2}=\frac{-2.158(C E V)}{(P V T)(C P F R)}+6.832 \\
& c_{2}=\frac{1.8}{b_{2}-1}
\end{aligned}
$$

The total dimensionless cough flow rate is the sum of Eqs. 10 and $11\left(\Theta=\Theta_{1}+\Theta_{2}\right)$. Using the above modeling, one can predict the cough characteristics from Eqs. 2-7, determine the Gamma probability model constants, and calculate the total dimensionless cough flow rate by integrating and summing Eqs. 10 and 11 as follows:

$$
\Theta=\frac{a_{1}}{\Gamma\left(b_{1}\right) c_{1}^{b_{1}}} \int_{0}^{1.2} \tau^{b_{1}-1} e^{\left(-\tau / c_{1}\right)}+\frac{a_{2}}{\Gamma\left(b_{2}\right) c_{2}^{b_{2}}} \int_{1.2}^{\infty}(\tau-1.2)^{b_{2}-1} e^{\left[-(\tau-1.2) / c_{2}\right]}
$$

The actual flow rate is determined from Eq. 16 as

$$
q_{g}=\Theta \times C P F R
$$

where $q_{g}=$ actual cough flow rate in liters/s.

\section{Droplet Fallout Model}

Based on the proposed physical model of expiratory function, a simplified mathematical model is proposed to predict the respiratory droplets contamination range before falling-out from a cough stream. To simplify the complexity of cough flow and droplets behavior, several assumptions are made. First, it is assumed that the cough is a steady state, one-dimensional, turbulent flow. Steady state flow is a plausible assumption according to Xie et al. (2007), which showed that the transient cough could be approximated as a steady jet. In addition, the Wei and Li (2017) experimental comparison of real cough with steady jet and sinusoidal cough velocity profiles showed that the real cough and simulated sinusoidal cough have a slightly higher droplets contamination range than the steady jet simulated cough. However, Rim and Novoselac (2008) compared particle transport in short transient and steady jets and found the latter has a higher droplets contamination range. In spite of the experimental findings of vortices (2D flow) in a cough stream by Tang et al. (2009) and Bourouiba et al. (2014), Hunt et al. (2007) found that vortices are negligible in steady jet flow as opposed to a transient jet. Bourouiba et al. (2014) and Bourouiba (2020) experimental and theoretical studies showed that coughing is a multiphase turbulent flow, which includes suspended large droplets and aerosol. Based on the above studies, steady state, 1D, and turbulent cough flow is assumed in this study. 
Second, it is assumed that liquid droplets and a cough air stream are in counter-current flow to enable the application of free-fall droplet terminal velocity. In actual cough flow, the liquid droplet downward velocity is perpendicular to the horizontal ejected airflow, resulting in a proportional (not exact) relationship between gas velocity and droplet velocity. This finding not only justifies the assumption of counter-current flow, but also gives a more conservative value of terminal velocity and thus droplets contamination range. Thirdly, the assumption of a rigid liquid droplet is based on the Brodkey (1967) experimental study on the relationship between droplet terminal velocity and droplet size. In his study, Brodkey found that a droplet size less than $1.7 \mathrm{~mm}$ in diameter behaves as rigid spheres, i.e. do not deform or distort, and follows the theoretical rigid sphere terminal velocity model. According to the reported droplet size of cough in Table 1, liquid droplet in a cough can be assumed rigid and spherical. Lastly, the constant liquid droplet size assumption, i.e. no droplet evaporation, is valid since this study is focused on large respiratory droplets $(\geq 5 \mu \mathrm{m})$, which behave in a ballistic manner.

Liquid droplet dynamics in a cough or sneeze are governed by three forces, namely gravitational, buoyancy, and drag force as shown in Fig. 8. A counter current droplet terminal velocity model assuming steady state flow conditions was derived from a force balance between vertical downward gravity force and upward vertical buoyancy and drag forces $\left(F_{g}=F_{b}+F_{d}\right)$ derived from momentum and mass balances. Assuming a rigid spherical liquid droplet, the force balance acting on a liquid droplet is given as follows:

$$
(1 / 6) \pi d_{d}^{3} g \rho_{L}=(1 / 6) \pi d_{d}^{3} g \rho_{g}+\frac{\pi C_{D} d_{d}^{2} \rho_{g} v_{d}^{2}}{8}
$$

Simplifying and solving Eq. 17 for the liquid droplet settling velocity gives the following expression:

$$
v_{d}=0.87\left[\frac{d_{d}\left(\rho_{L}-\rho_{g}\right) g}{C_{D} \rho_{g}}\right]^{0.5}
$$

where $C_{D}=$ dimensionless drag coefficient, $d_{d}=$ droplet diameter in $\mathrm{m}, v_{d}=$ droplet terminal velocity in $\mathrm{m} / \mathrm{s}, \rho_{L}$ $=$ liquid density in $\mathrm{kg} / \mathrm{m}^{3}, \rho_{g}=$ gas density in $\mathrm{kg} / \mathrm{m}^{3}$, and $g=$ gravity acceleration in $\mathrm{m} / \mathrm{s}^{2}$. The dimensionless drag coefficient has various forms depending on the flow regime. For example, for creeping flow $\left(N_{R e}<0.1\right)$, the drag coefficient is derived from the mass and momentum conservation laws and is a function of Reynolds number as $C_{D}=24 / N_{R e}$, where Reynolds number is $N_{R e}=\rho v d_{d} / \mu$. For an intermediate range of Reynolds number $\left(2<N_{R e}<5 \times 10^{2}\right)$, the drag coefficient is empirically derived from experimental data as $C_{D}=18.5 / N_{R e}{ }^{3 / 5}$. For a higher range of Reynolds number $\left(5 \times 10^{2}<N_{R e}<2 \times 10^{5}\right)$, i.e. turbulent flow, the drag coefficient reaches a constant value of 0.44 . In this study, and due to cough being in the turbulent flow regime (Bourouiba, 2020), the 0.44 drag coefficient is used as an input to the model. 


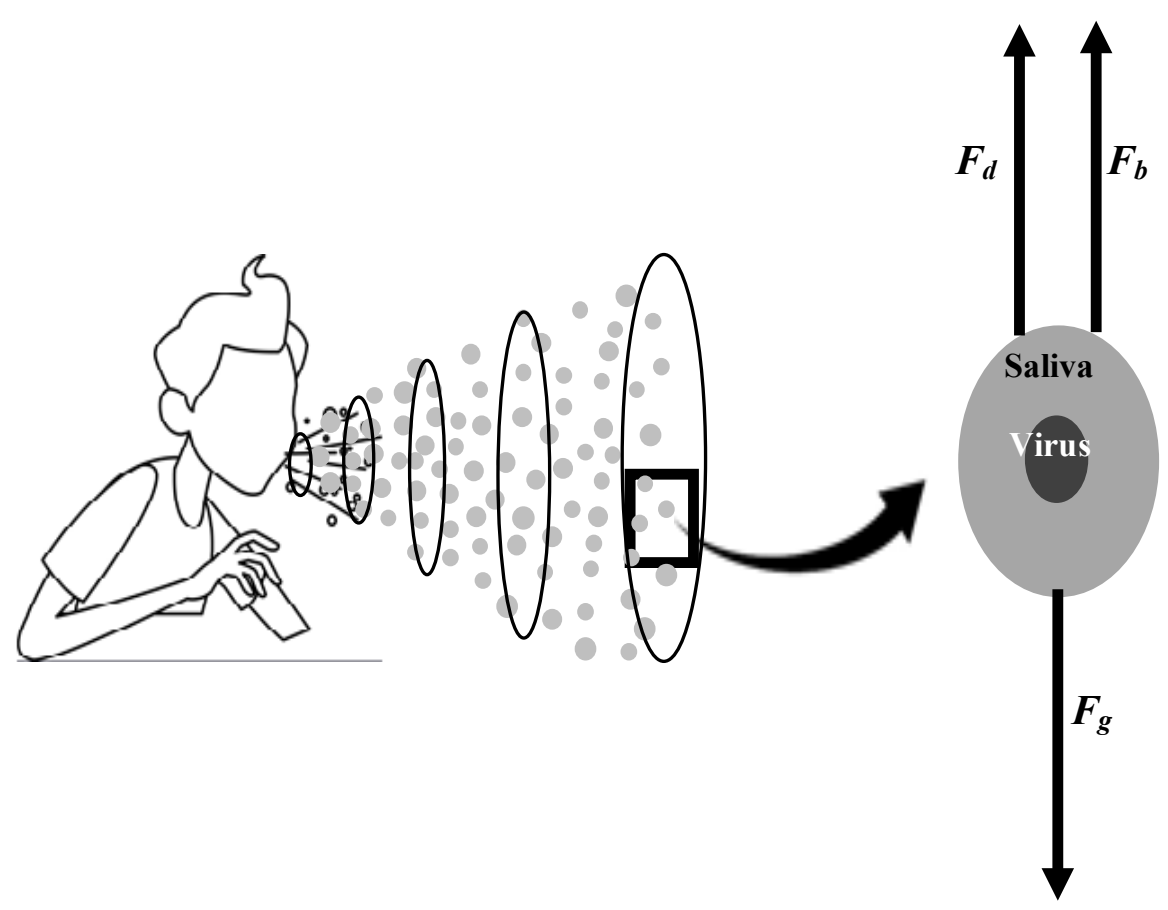

Figure 8. Forces acting on liquid mucosalivary droplet during a cough.

The gas velocity in the cough stream varies with respect to the distance from the source because of the increasing cough flow cross-sectional area as shown in Fig. 8. Thus, the actual gas velocity in term of distance (droplets contamination range) is given as follows:

$$
v_{g}=\frac{q_{g}}{A_{c}}
$$

where $v_{g}=$ cough gas velocity in $\mathrm{m} / \mathrm{s}, q_{g}=$ cough flow rate in $\mathrm{m}^{3} / \mathrm{s}$, and $A_{c}=$ cough cross-sectional area in $\mathrm{m}^{2}$. The cough flow $\left(q_{g}\right)$ rate and the cough cross-sectional area $A_{c}$ are given in Eqs. 16 and 1, respectively. The condition for a liquid droplet to fall out from a cough cloud is when the cough gas velocity is equal to the droplet settling velocity $\left(v_{d}=v_{g}\right)$. Therefore, equating Eqs. 18 and 19 yields the minimum horizontal distance (contamination range) $\mathrm{D}$ at which droplets fall out from the cough stream. An iterative calculation scheme is required to calculate the droplets contamination range. Fig. 9 shows a flow chart of the calculation procedure.

\section{RESULTS AND DISCUSSION}

The above-proposed model provides insights to the relationship between droplets contamination range of exhaled mucosalivary droplets and several important parameters, such as cough flow rate, saliva droplet size, saliva drag coefficient, and liquid and gas densities. A sensitivity analysis was carried out in this study to investigate the effect of cough flow rate (gender and adult/child), saliva droplet size, and drag coefficient on the droplets contamination range to improve the understanding of what governs the droplets contamination range to better design a mitigation plan and social distancing. 


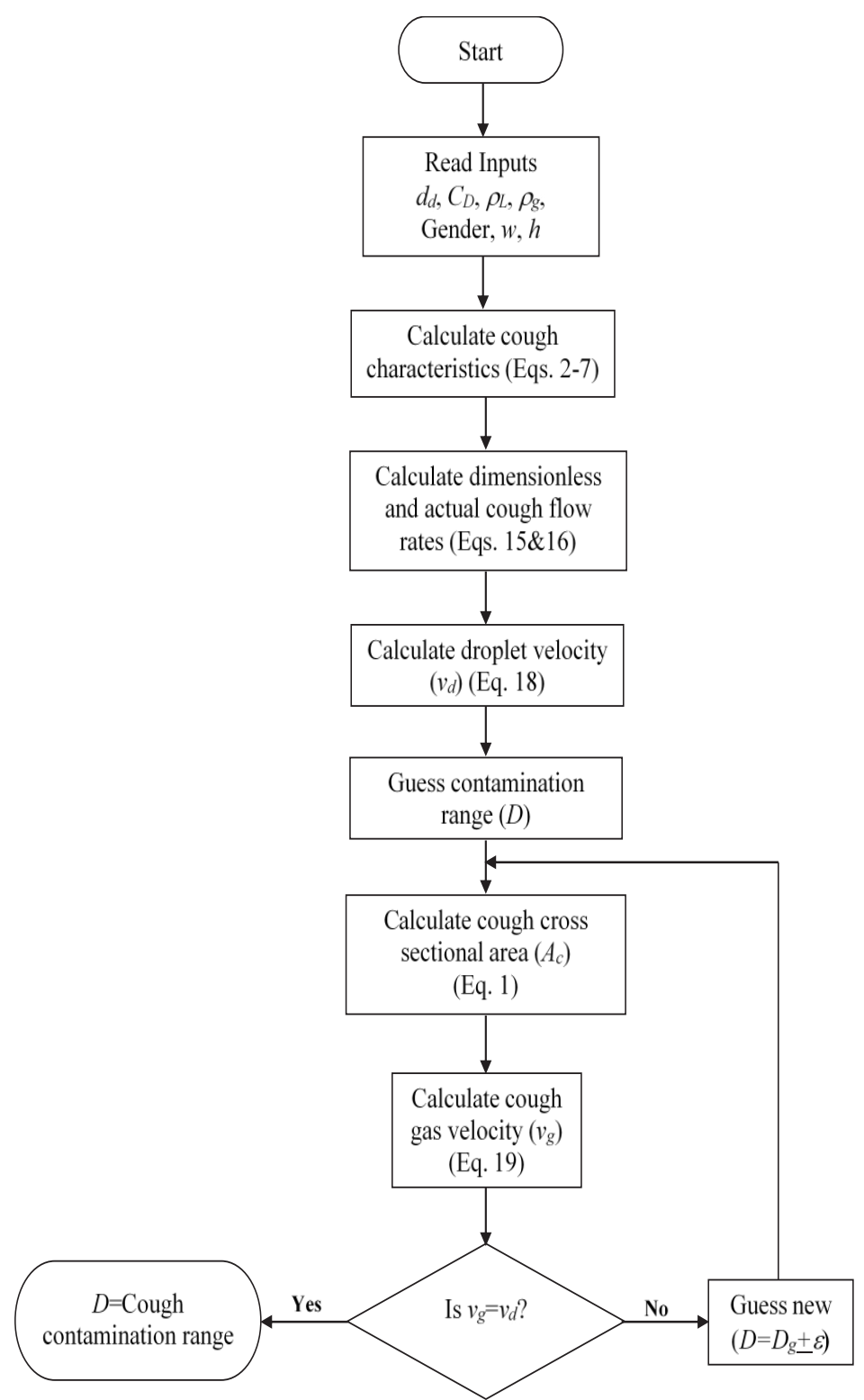

Figure 9. Iterative calculation procedure of the cough droplets contamination range.

To predict the droplets contamination range of a cough, the proposed mathematical model presented in the previous section was coded and executed. A base case of typical input parameters ( $d_{d}=16$ microns, $\rho_{L}=1100 \mathrm{~kg} / \mathrm{m}^{3}$, $\rho_{g}=1.2 \mathrm{~kg} / \mathrm{m}^{3}, C_{D}=0.44$, male, $w=80 \mathrm{~kg}, h=1.7 \mathrm{~m}$ ) was simulated, resulting in a droplets contamination range of an adult male cough of approximately 2.3 meters. To investigate the effects of gender and age (adult vs. child), a similar simulation run was carried out for a female and a child, predicting a cough droplets contamination range of $1.89 \mathrm{~m}$, and $1.2 \mathrm{~m}$, respectively, as shown in Fig. 10. The reason for the different droplets contamination ranges of male, female, and child is the different cough flow rate of males and females, which depends on the weight and height as given by the Gupta et al. (2009) model (Eqs. 2-7). This dependency of cough flow rate on gender, weight, and height of human beings is related to the lung size and air capacity variation with gender and body size. The above predicted 
droplets contamination range is within the recommended person-to-person social distance of 1.5-2 meters (CDC, 2020). In addition, Xie et al. (2007) found that droplets generated by cough could travel 2 meters, and by sneeze 6 meters. It is important to keep in mind that this is the minimum safe distance, as it is sensitive to droplet size and other environmental conditions such as temperature and relative humidity. This distance is also reasonable and a compromise between the social and communication needs and scientific findings.

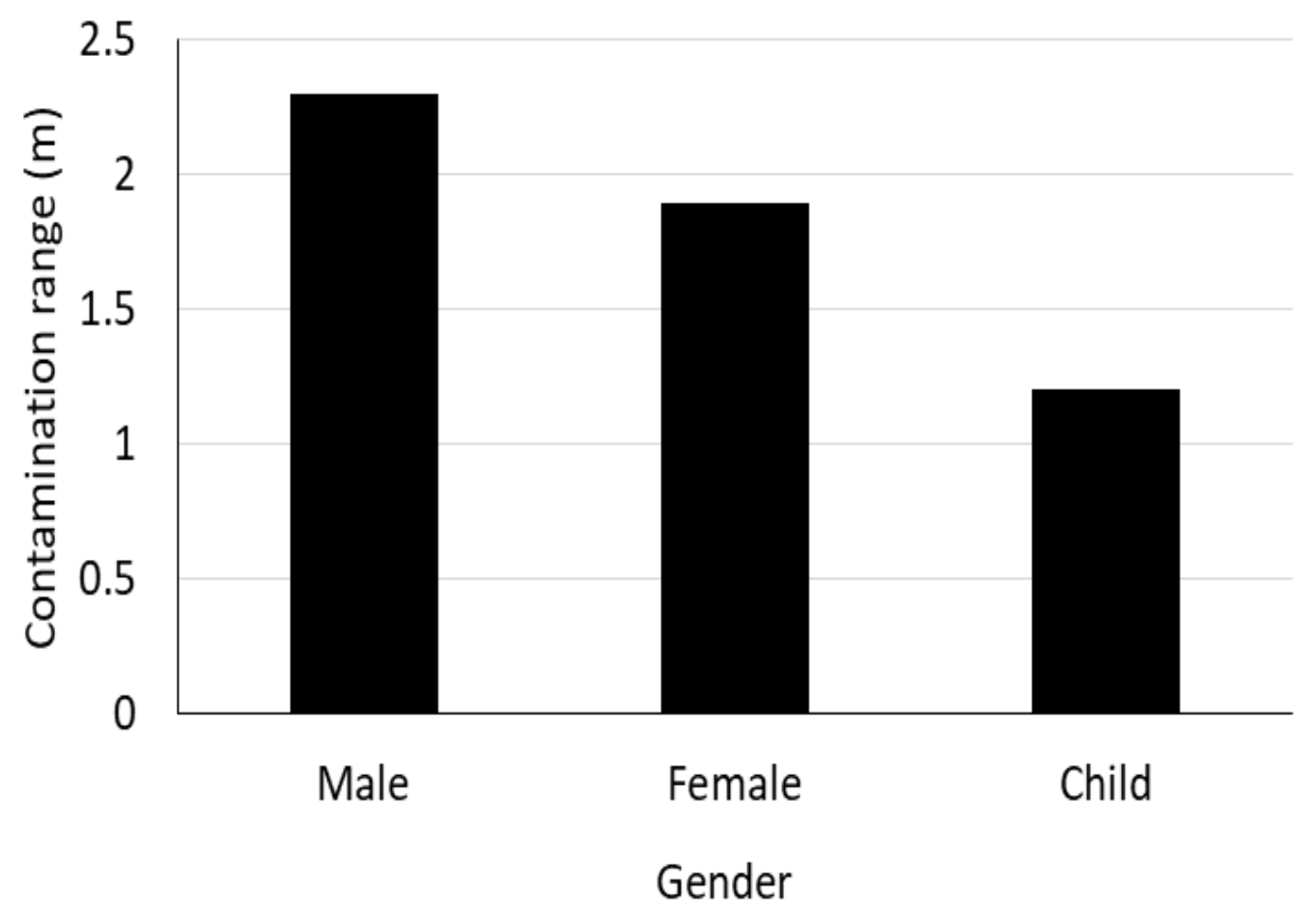

Figure 10. Model prediction of cough droplets contamination range $\left(d_{d}=16\right.$ microns, $\rho_{L}=1100 \mathrm{~kg} / \mathrm{m}^{3}, \rho_{g}=1.2 \mathrm{~kg} / \mathrm{m}^{3}$, $C_{D}=0.44$, male and female: $w=80 \mathrm{~kg}, h=1.7 \mathrm{~m}$, child: $w=32 \mathrm{~kg}, h=1.4 \mathrm{~m}$ ).

The effect of the exhaled saliva droplet size during a cough on droplets contamination range was investigated in this study as well. Fig. 11 shows that as droplet size increases, the cough droplets contamination range decreases for both genders. Droplet size varies with the type of respiratory function as shown in Table 1. In addition, the effect of droplet drag coefficient $\left(C_{D}\right)$ was found to be significant in this study, as shown in Fig. 12. The cough droplets contamination range increases as the drag coefficient increases for both genders. The importance of this relationship relies in the dependency of drag coefficient on ambient air viscosity, which increases as the ambient temperature increases. This indicates that as the ambient temperature increases, i.e. during summer season, ambient air viscosity increases, resulting in higher drag coefficient and larger cough droplets contamination range. This finding suggest that greater inter-personal distance should be maintained during the summer season to mitigate COVID-19, especially in closed areas. In addition, the ambient temperature may promote the transmission of COVID-19 by evaporating the large droplets into aerosol, which stay suspended in air for a long time and can travel a long distance with the airflow and ventilation system. 


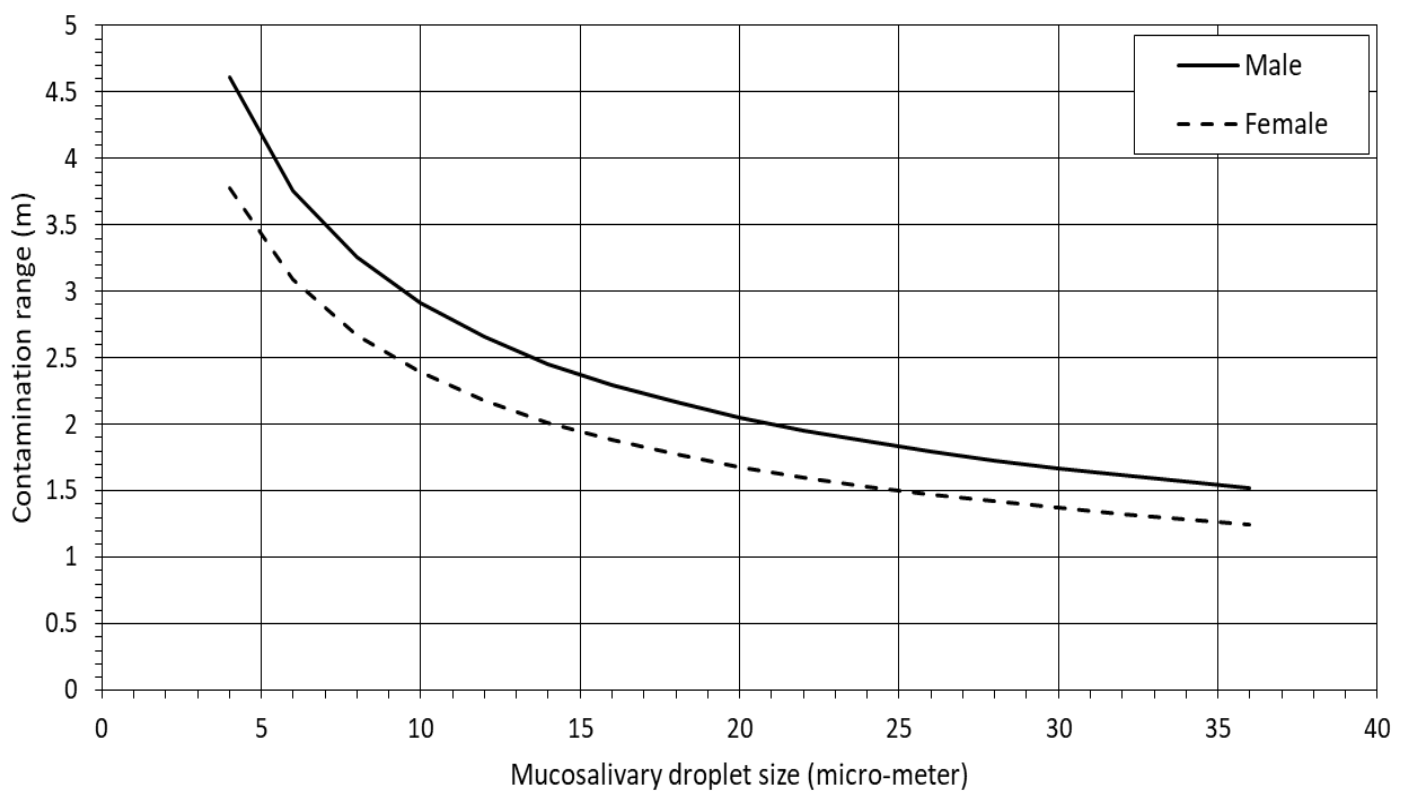

Figure 11. Predicted effect of expelled droplet size on cough droplets contamination range $\left(\rho_{L}=1100 \mathrm{~kg} / \mathrm{m}^{3}\right.$, $\rho_{g}=1.2 \mathrm{~kg} / \mathrm{m}^{3}, C_{D}=0.44$, male and female: $\left.w=80 \mathrm{~kg}, h=1.7 \mathrm{~m}\right)$.

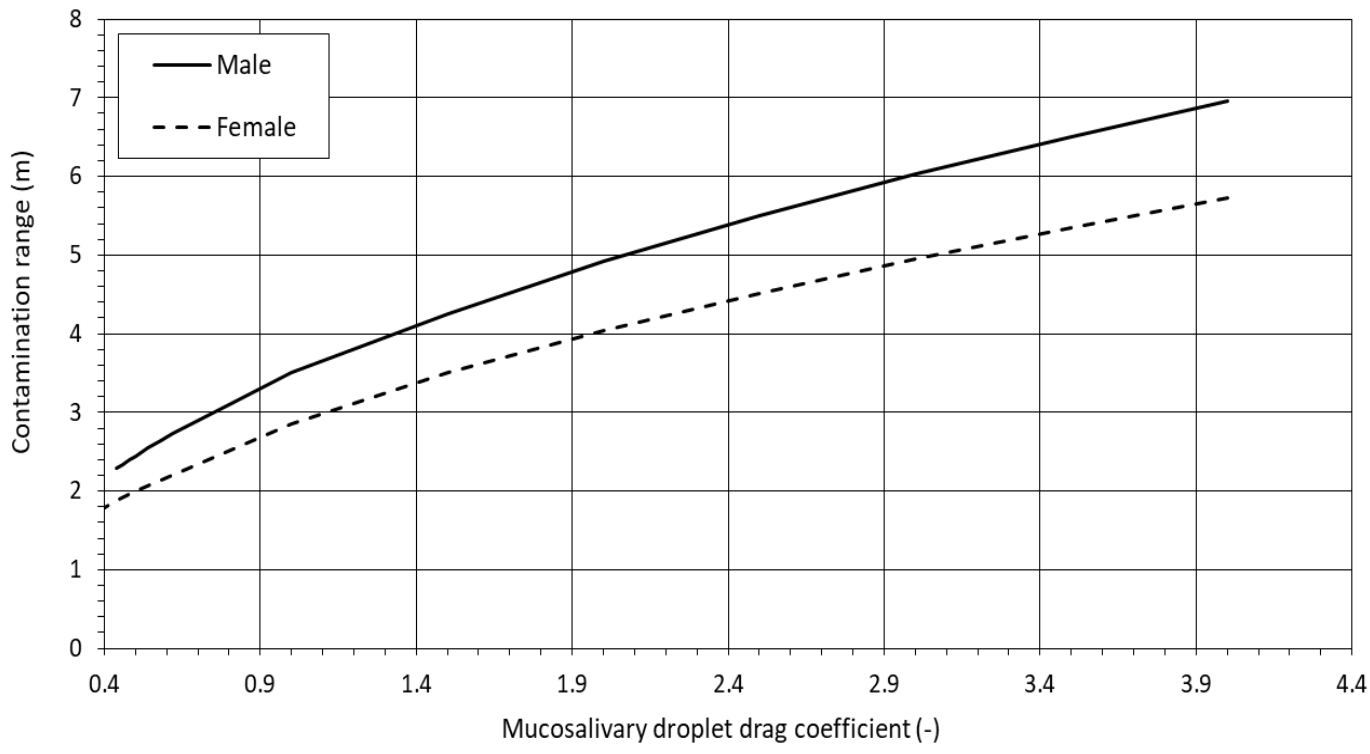

Figure 12. Predicted effect of droplet drag coefficient on cough droplets contamination range $\left(d_{d}=16\right.$ microns, $\rho_{L}=1100 \mathrm{~kg} / \mathrm{m}^{3}, \rho_{g}=1.2 \mathrm{~kg} / \mathrm{m}^{3}$, male and female: $\left.w=80 \mathrm{~kg}, h=1.7 \mathrm{~m}\right)$.

Another ambient condition that affects the air viscosity (and drag coefficient) is the relative humidity. As opposed to temperature, as relative humidity increases, air viscosity decreases, resulting in lower drag coefficient and 
lower droplets contamination range. This information is significant to better mitigating COVID-19 as air humidity increases. Simulation runs showed that the effect of the expelled gas and droplet densities on the contamination range during a cough is negligible. The density of the gas was found to be slightly directly proportional, while the droplet density is slightly inversely proportional.

\section{CONCLUSION}

A mathematical model to predict cough droplets contamination range is proposed in this study to estimate the required social distance to mitigate COVID-19 transmission. The mathematical model is based on a proposed simple physical model postulated from available experimental observations and measurement results. The model predicted a cough droplets contamination range of 2.3 meter for adult males using an average droplet size of 16 microns. A sensitivity analysis was carried out to investigate the effects of gender and age (adult vs. child), ejected saliva droplet size (different respiratory functions), and droplet drag coefficient, which is related to ambient temperature and relative humidity (i.e. season of the year). Results show that adult males require greater social distance than adult females or children due to the greater exhaled air flowrate (larger lung size and air capacity). A significant effect of ejected saliva droplet size on the contamination range was predicted, which shows that as droplet size increases, the contamination range decreases, indicating that a greater social distance is required for other respiratory functions, in which the droplet size is smaller. Furthermore, the effect of surrounding air drag coefficient acting on ejected saliva droplets was found to be significant with a direct proportional nonlinear relationship. The drag coefficient is related to air viscosity, which is a function of ambient temperature, indicating that as ambient temperature increases, for example during summer season, greater person-to-person distance is required to mitigate the transmission of COVID-19. Conversely, as relative humidity increases, air viscosity decreases, resulting in lower drag coefficient and lower droplets contamination range. In conclusion, the proposed simple mathematical model provides deeper understanding and reasonable predictions of cough droplets contamination range, which can be used to set guidelines for social distancing to mitigate COVID-19. The proposed physical and mathematical models provide a reasonable framework and serves as a foundation for further experimental and modeling studies.

\section{REFERENCES}

Aliabadi, A., Rogak, S., Bartlett, K. et al. 2011. Preventing Airborne Disease Transmission: Review of Methods for Ventilation Design in Health Care Facilities. Advances in Preventive Medicine, vol. 2011, Article ID 124064, 21 pages. doi:10.4061/2011/124064.

Asadi, S., Bouvier, N., Wexler, A. et al. 2020. The Coronavirus Pandemic and Aerosols: Does COVID-19 Transmit Via Expiratory Particle? Aerosol Sci. and Tech., 54:6, 635-638.

Asadi, S., Wexler, A., Cappa, C. et al. 2019. Aerosol Emission and Super-emission during Human Speech Increase with Voice Loudness. Science Rep. 9 (1), 2348.

Bourouiba, L. 2020. Turbulent Gas Clouds and Respiratory Pathogen Emissions - Potential Implications for Reducing Transmission of COVID-19. Clinical Review and Education, American Medical Association (https://jamanetwork.com).

Bourouiba, L., Dehandschoewercker, E., and Bush, J. 2014. Violent Expiratory Events: On Coughing and Sneezing. J. Fluid Mech. 745, 537-563.

Brodkey, R. 1967. The Phenomena of Fluid Motion. Reading, Massachusetts, USA: Addison, Wesley.

CDC, US Centers for Disease Control 2020. https://www.cdc.gov/coronavirus/2019-ncov/prevent-gettingsick/prevention.html. 
Chao, C., Wa, M., Morawska, L. et al. 2009. Characterization of Expiration Air Jets and Droplet Size Distribution Immediately at the Mouth Opening. Journal of Aerosol Science, vol. 40, no. 2, pp. 122-133.

Drossinos, Y., and Stilianakis, N. 2020. What Aerosol Physics tells us about Airborne Pathogen Transmission? Aerosol Science and Technology, 54:6, 639-643. https://doi.org/101080/02786826.2020.1751055.

Duguid, J. 1946. The Size and the Duration of Air-Carriage of Respiratory Droplets and Droplet-Nuclei. The journal of Hygiene, vol. 44, no. 6, pp. 471-479.

Gupta, J., Lin, C., and Chen, Q. 2009. Flow Dynamics and Characterization of a Cough. Indoor Air, 19, 517 525.

Hsiao, T., Chuang, H., Griffith, S., Chen, S., Young, L., 2020. COVID-19: An Aerosol's Point of View from Expiration to Transmission to Viral-mechanism. Aerosol and Air Quality Research, 20: 905-910. doi: 10.4209/aaqr.2020.04.0154.

Hunt, J., Delfos, R., Eames, I. et al. 2007. Vortices, Complex Flows and Inertial Particles. Flow Turbulence Combust, 79: 207-234.

Lamb, J., Murty, G., Slater, R. et al. 1993. Postoperative Laryngeal Function Assessed by Tussomerty. British Jr. of Anesthesia, 70, 478-479.

Leiner, G., Abramowitz, S., Small, M. et al. 1966. Cough Peak Flow Rate. The American Jr. of the Medical Sci., 251 (2), 211-214.

Loudon, R., and Roberts, R. 1967. Relation between the Airborne Diameters of Respiratory Droplets and the Diameter of Respiratory Droplets and the Diameter of the Stains Left after Recovery. Nature, vol. 213, no. 5071, pp. 95-96.

Mahajan, R. Singh, P., Murty, G. et al. 1994. Relationship between Expired Lung Volume, Peak Flow Rate, and Peak Velocity Time during a Cough maneuver. British Jr. of Anesthesia, 71 (3), 298-301.

Mittal, R., Ni, R., and Seo, J-H. 2020. The Flow Physics of COVID-19. J. Fluid Mech., vol. 894, F2.

Papineni, R., and Rosenthal, F. 1997. The Size Distribution of Droplets in the Exhaled Breath of Healthy Human Subjects. Journal of Aerosol Medicine, vol. 10, no. 2, pp. 105-116.

Qamar, M. 2020. COVID-19: A Look into the Modern Age Pandemic. Journal of Public Health: from Theory to Practice. https://doi.org/10.1007/s10389-020-01294-z.

Rim, D. and Novoselac, A. 2008. Transient Simulation of Airflow and Pollutant Dispersion under Mixing Flow and Buoyancy Driven Flow Regimes in Residential Buildings. ASHRAE Transactions, 114(2): 130-142.

Singh, P., Mahajan, R., Murty, G. et al. 1995. Full-Scale Schlieren Flow Visualization. Flow Visualization VII, ed. Crowder, J., Begell House, New York, 2-13.

Tang, J., Liebner, T., Craven, B. et al. 2009. A Schlieren Optical Study of the Human Cough with and without Wearing Masks for Aerosol Infection Control. Jr. of Royal Society Interface, 6 (Suppl 6): S727-S736.

Walker P., Whittaker C., Watson, O., Baguelin, M., Ainslie, K., Bhatia, S. et al. 2020. The Global Impact of COVID-19 and Strategies for Mitigation and Suppression. WHO Collaborating Center for Infectious Disease Modeling, MRC Center for Global Infectious Disease Analysis, Abdul Latif Jameel Institute for Disease and Emergency Analytics, Imperial College London.

Weber, T., and Stilianakis, N. 2008. Inactivation of Influenza A viruses in the Environment and Modes of Transmission: A critical Review. J. Infect. 57 (5): 361-373. Doi: 10.1016/j.jinf.2008.08.013.

Wei, J., and Li, Y. 2017. Human Cough as a Two-Stage Jet and Its Role in Particle Transport. PLoS ONE 12(1):e0169235. Doi:10.1371/journal.pone.0169235.

World Health Organization (WHO) 2020. Coronavirus Disease 2019 (COVID-19): Situation Report - 66, World Health Organization. 
310 Application of multiphase flow and droplet separation theory in modeling cough droplets contamination range to mitigate COVID-19 transmission: Do not stand too close to me!

Xie, X., Li, Y., Chwang, A. et al. 2007. How Far Droplets Can Move in Indoor Environment - Revisiting the Wells Evaporation - Falling Curve. Indoor Air 17(3): 211-225. Doi: 10.1111/j.1600-0668.2007.00469.x PMID: 17542834.

Zhao, B., Zhang, Z., Li, Z. 2005. Numerical Study of Transport of Droplets or Particles Generated by Respiratory System Indoors. Building and Environment Jr., 40 (8), 1032-1039.

Zhu, S., Kato, S., Yang, J. 2006. Study on Transport Characteristics of Saliva Droplets Produced by Coughing in a Calm Indoor Environment. Building and Environment Jr., 41, 1691-1702. 



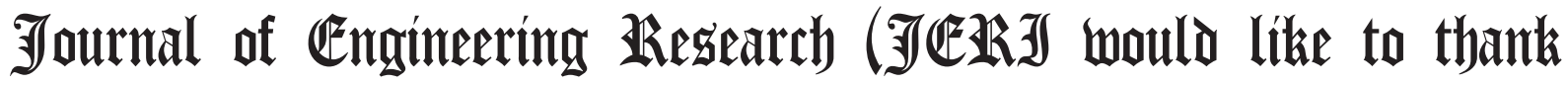
the distinguished indibiduals who put an appreciated effort in refereeing the papers publisted in this issue. Bour valuable time and imput is biathly recoumised by the editorial board of $\mathfrak{J} \mathbb{E}$ R, we look forward to more cooperation in the future to kerp $\mathfrak{J}$ e 

